\title{
Comparison between Z-plasty and V-Y Advancement for the Surgical Correction of Cryptotia
}

Young Kyoo Cho, Sung Gun Bae, Byung Chae Cho

Department of Plastic and Reconstructive Surgery, Kyungpook National University Hospital, Kyungpook National University School of Medicine, Daegu, Korea

No potential conflict of interest relevant to this article was reported.

\begin{abstract}
Background: Cryptotia correction by $\mathrm{V}-\mathrm{Y}$ advancement of a temporal triangular flap was introduced in 2005. However, despite the several advantages of V-Y advancement, visible scars at the donor site are problematic. As a result, a Z-plasty technique was considered for skin deficiency in mild cases. Therefore, we introduce a new surgical scheme for cryptotia correction based on considerations of techniques and complications that arose in our clinic.

Methods: Between 2000 and 2013, 26 patients (35 cases) of cryptotia were treated. Seventeen patients had unilateral cryptotia and nine had bilateral cryptotia. Two corrective methods were used, Z-plasty or V-Y advancement, based on the severity. In mild cases, Z-plasty was used for correction and in severe cases, V-Y flap advancement was used for more skin supplement.

Results: Follow-up periods ranged from 6 months to 1.5 years. The results obtained were relatively favorable. Nine cases of mild deformity were corrected by Z-plasty, and the other 26 cases with mild or severe deformities were corrected by V-Y advancement. In Z-plasty cases, there was one hypertrophic scar and in $\mathrm{V}-\mathrm{Y}$ advancement cases, seven resulted in visible scarring and three in skin sloughing.

Conclusion: The main advantage of Z-plasty is a lower likelihood of visible scarring at the donor site. In mild cases, Z-plasty may be a good alternative, but in severe cases, $\mathrm{V}-\mathrm{Y}$ advancement is probably the best option for more skin supplement.
\end{abstract}

Keywords: Cryptotia / V-Y advancement / Z-plasty

\section{INTRODUCTION}

Cryptotia is a relatively common congenital auricular deformity in Asians [1]. Its main abnormalities were the burying of the upper pole of the ear cartilage beneath the skin of the scalp and obliteration of the auriculocephalic sulcus. In addition, cryptotia is often accompanied by deformity of the antihelix, especially its superior crus. The Hirose's classification is widely used to classify cryptotia.

\footnotetext{
Correspondence: Byung Chae Cho

Department of Plastic and Reconstructive Surgery, Kyungpook National University Hospital, Kyungpook National University School of Medicine, 130 Dongdeok-ro, Jung-gu, Daegu 700-721, Korea

E-mail: bccho@knu.ac.kr

*This research was supported by the Kyungpook National University Research Fund, 2012.

Received February 12, 2014 / Revised March 3, 2014 / Accepted March 10, 2014
}

This classification is based on the type of cartilage constriction caused by abnormally developed intrinsic muscle. Type I is the transverse muscle type or superior crus type and type II is the oblique muscle type or inferior crus type [2].

Treatment goals can be summarized as follows; 1) restoration of the auriculocephalic sulcus, 2) replacing of the deficient skin flap to cover embedded cartilage, 3) establishing collapsed cartilage, and 4) releasing abnormal intrinsic auricular muscles, all while minimizing donor site morbidity $[3,4]$.

Various operative techniques have been described, such as V-Y plasty, Z-plasty, local skin flaps, and various combinations of flaps and skin grafts, and relatively favorable results have been routinely achieved [5]. However, several problems remain, such as alopecia, visible scars, undercorrected auriculotemporal sulcus, and in- 
complete correction of auricular cartilage. That is to say, each method has its advantages and disadvantages with respect to skin flap deficiencies. We have adopted two methods, Z-plasty or V-Y advancement, based on considerations of cryptotia severity. In mild cases, Z-plasty was used for correction, and in severe cases, a V-Y flap advancement was used. The aim of this study is to compare the merits and demerits of these two surgical techniques for the correction of cryptotia and can be helpful to choose proper method for each case.

\section{METHODS}

Between 2000 and 2013, 26 patients with cryptotia were treated in our clinic. Patient ages ranged from 3 to 23 years, and there were 22 males and 4 females. Seventeen patients had unilateral cryptotia and 9 had bilateral cryptotia. In the 17 patients with unilateral cryptotia, 3 had left side cryptotia and 14 had right side cryptotia. Hirose et al. [2] classified cryptotia into two types depending on the intrinsic muscle anomaly and antihelical deformity. Type I cryptotia is classified as a transverse muscle type located on the superior crus of the antihelix. This group has a broadened transverse intrinsic muscle which bends the antihelix. Type II cryptotia is described as an oblique muscle type. In this group, the oblique muscle abnormality is inserted in the inferior crus, bending the inferior crus anteriorly. A sharply curved antihelix by severe compression of the body of the antihelix and the superior crus (type I) presented in 23 cases, severe contracture of superior crus presented a third crus (type I) in 6 cases, and adhesion of the helix cartilage to the scaphoid cavity with severe contracted inferior crus of the antihelix (type II) presented in 6 cases. Among 29 cases in type I, 18 cases were mild deformities and 11 cases were severe deformities. Among 6 cases in type II, 2 cases were mild deformities and 4 cases were severe deformities.

\section{Operative techniques}

\section{Z-plasty (modified Onizuka's method)}

The correction of cryptotia using Z-plasty was designed on the periauricular area with extension to the hair-bearing scalp or not, using a modification of Onizuka's method [5-7]. Traction is applied to the upper part of the auricle away from the scalp, and two flaps (a small anterior based flap and a large posterior based flap) are designed along the auricular sulcus. The lateral limb of the anterior based flap is drawn along the buried portion of the superior helix. The lateral limb of posterior based flap is drawn along the hairline extending the point 2 to $3 \mathrm{~cm}$ superoanterior from the helical root to below the midlevel of the auricle. If needed, a back cut is performed on inferior edge of the lateral arm of the posterior based flap. The central limb begins at the apex of the buried portion forming the superior helix and runs across the point superoanterior direction from helical root. The width of two flaps is about 2 to $2.5 \mathrm{~cm}$ avoiding interruption in the flap circulation. The two flaps are elevated in the level below the subcutaneous fat layer (Fig. 1).

\section{V-Y advancement of a temporal triangular flap}

Cryptotia correction by V-Y advancement of a temporal triangular flap was introduced in 2005 [8,9]. The anterior margin of the triangular flap ends in the skin of the anterior part of the buried portion of the superior helix. The posterior margin of the flap is continued to the mastoid area 2 to $3 \mathrm{~cm}$ posterior to the posterior auricular sulcus and ends at the midlevel of the auricle. The lower part of the flap is modified into a curved shape of width 5 to $6 \mathrm{~cm}$ to provide sufficient skin flap on the upper portion of the auricle. The triangular flap is elevated from the temporal scalp above the superficial temporal fascia level, for the Z-plasty flap. The amount of skin replacement is greater in V-Y flap advancement than Zplasty. So, we performed V-Y flap advancement to replace sufficient volume of skin in severe case cryptotia (Fig. 2).

\section{Manipulation in upper part of the auricle}

After flap elevation, further dissection is performed immediately above the perichondrium on the upper part of the auricle, especially its posterior surface. Deformity is due to abnormal muscle insertion, for example, of the superior auricular muscle or the intrinsic transverse muscle. Therefore, abnormal insertion of the superior auricular muscle should be released from the upper part of the helix. After the posterior surface of the upper auricle is ex- 


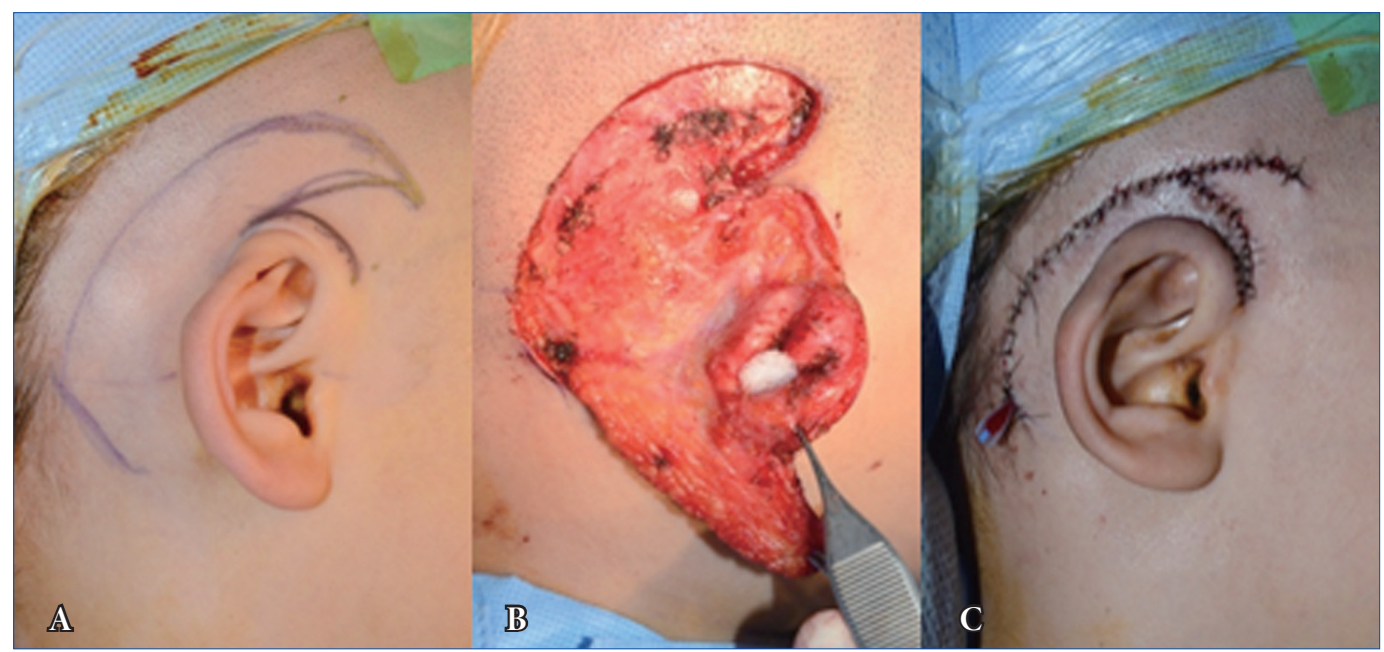

Fig. 1. Operative technique of Z-plasty. (A) Preoperative design. (B) Dissection of muscle at the posterior surface of antihelical cartilage and fibrous tissue to release contracture of the antihelix. Fixation of Medpor to the posterior aspect of the corrected and sharply curved antihelical fold. (C) Immediate postoperative view.

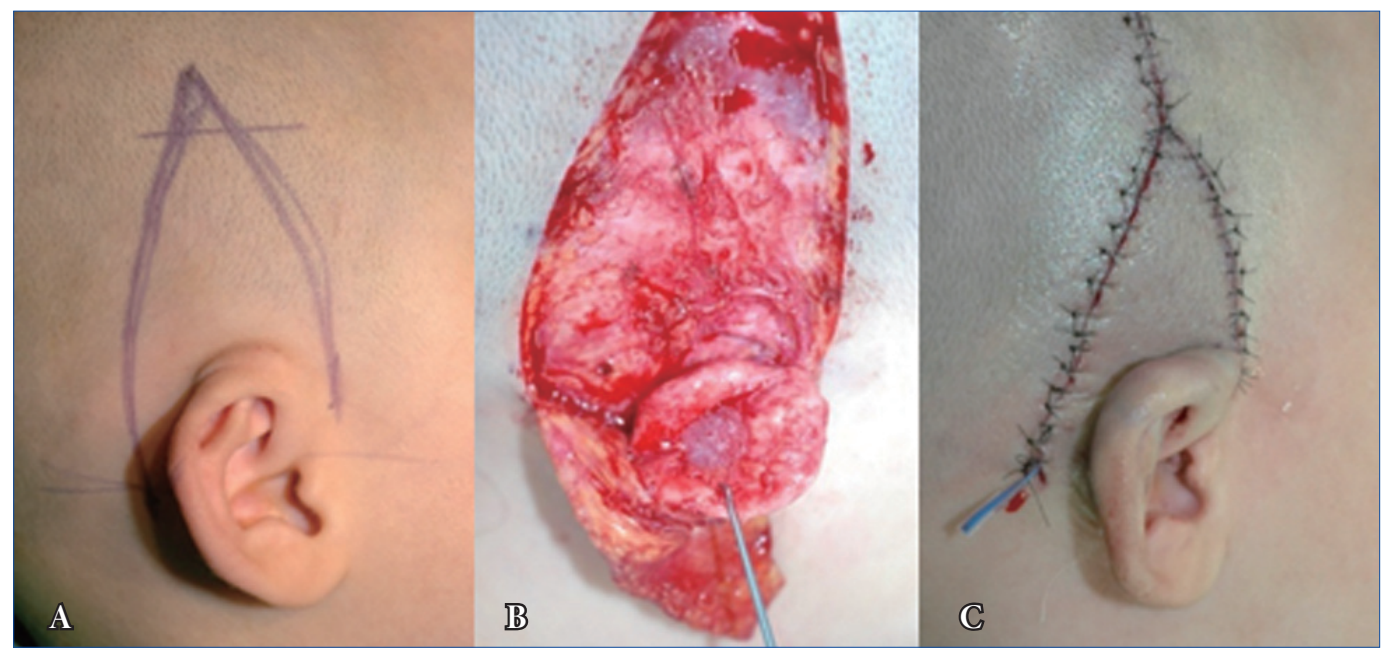

Fig. 2. Operative technique of V-Y flap advancement. (A) Preoperative design. (B) Dissection of abnormal muscle and fibrous tissue of the antihelix. Fixation of Medpor to the posterior aspect of the corrected and sharply curved antihelical fold. (C) Immediate postoperative view.

posed completely, the muscle at the posterior surface of the antihelical cartilage and fibrous tissue are dissected to release the contracture of the antihelix. In cases with a sharply curved antihelical crus, correction is done by multiple scoring on the posterior aspect and strut of a Medpor sheet or autogenous cartilage. Considering the deformity and contraction of auricular cartilage, conchal cartilage $0.7 \times 0.7 \mathrm{~cm}$ size in mild cases, a thin $0.85-\mathrm{mm}$ Medpor sheet (Medpor Surgical Implant, Porex Surgical Inc., College Park, GA, USA) $0.5 \times 1.5 \mathrm{~cm}$ size in severe cases is inserted and fixed with 5-0 nylon sutures to the posterior aspect of the cor- rected sharply curved antihelical crus for preventing relapse. If the cartilage of upper helix is severely contracted, Musgrave's technique is indicated for correction [10]. Contracted helical cartilage is fanned out upward and backward using radiating incisions and 6-0 white nylon mattress sutures. Gaps in the periphery of the helix as cartilage strips fan out are bridged using conchal cartilage graft harvested from the opposite auricle.

\section{Flap fixation and closure}

For Z-plasty, the two flaps are replaced on each other. The large 
posterior based flap is lowered to the caudal side and advanced in the anterior direction to cover the superior helix. The small anterior based flap is transposed to the cephalic side. For V-Y advancement, the triangular flap prepared in the upper auricular region is advanced inferiorly to prepare the upper auricular half.

To provide ample skin after flap replacement, the auricular sulcus is created using two key 5-0 white nylon sutures. The key sutures are done on the subcutaneous tissue of the flap and the cartilage of the auriculocephalic sulcus. Flap and donor site closure are performed with 4-0 Vicryl and 5-0 nylon. Occasionally, the dog-ear portion that develops at the helical root area should trimmed. In case of insufficient convolution, bolster fixations were used on upper part of helix for 2 or 3 days after the operation.

\section{RESULTS}

In total, 35 cases (26 patients) of cryptotia were treated. Follow-up periods ranged from 6 months to 1.5 years, with relatively favorable results (Figs. 3, 4). Nine cases with mild deformities were corrected by Z-plasty, and the other 26 with mild or severe deformities were corrected by V-Y advancement (Table 1). Considering
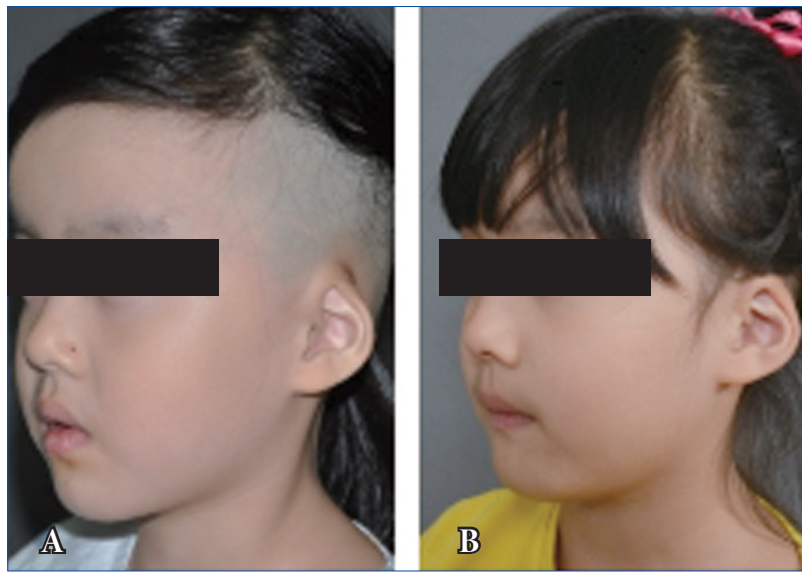

Fig. 3. A 6-year-old girl with cryptotia of the left auricle corrected by V-Y flap advancement. (A) Preoperative view. (B) Six-month postoperative view.

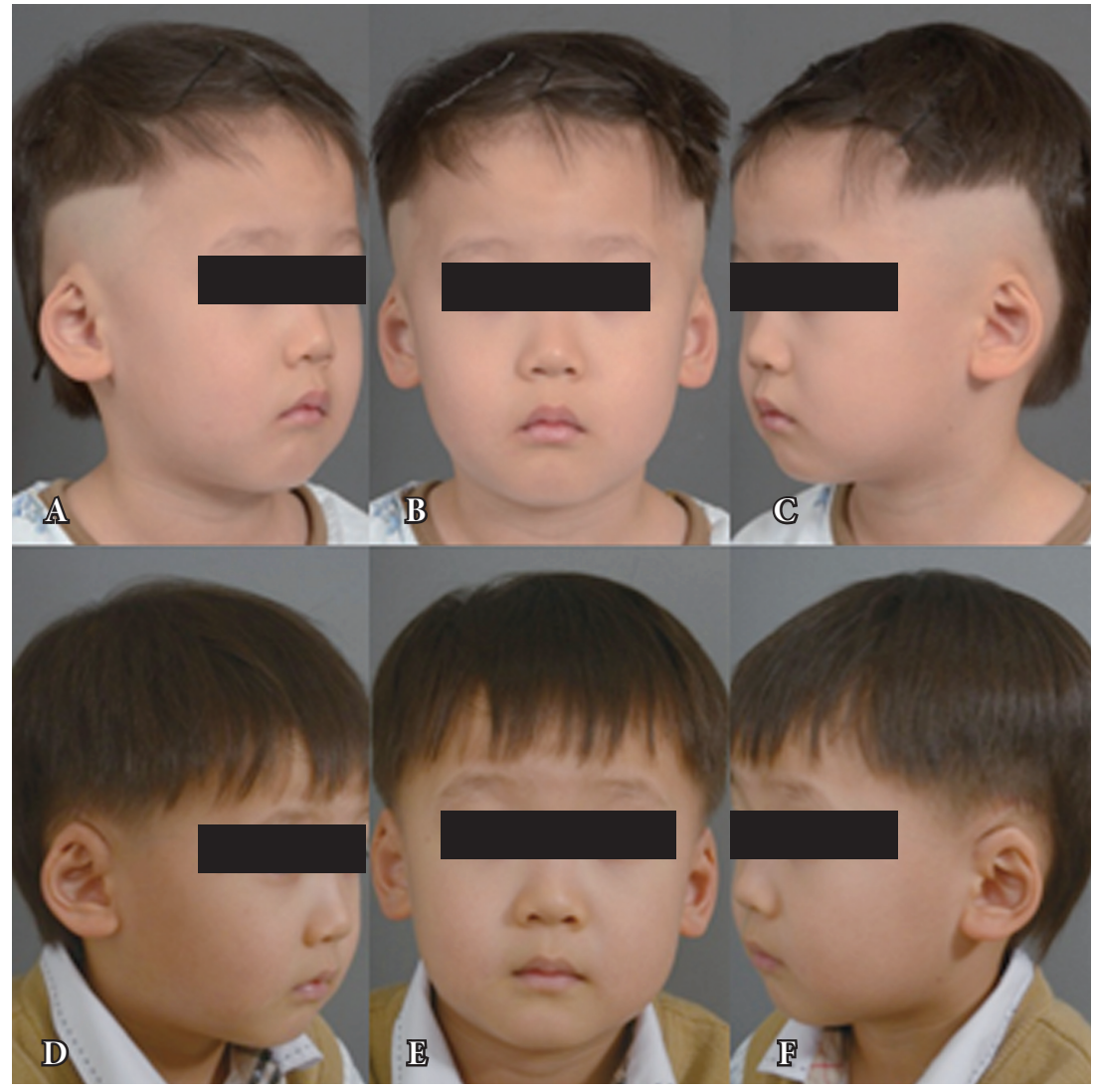

Fig. 4. A 3-year-old boy with bilateral cryptotia corrected by Z-plasty. (A-C) Preoperative view. (D-F) Six-month postoperative view. 
the deformity and contraction of auricular cartilage, conchal cartilage was used in mild cases, Medpor sheet was used in severe cases to the posterior aspect of the corrected sharply curved antihelical crus for preventing relapse as a splinting. No exposure of Medpor occurred. Complications after correction by Z-plasty or V-Y advancement were evaluated on early to delayed time (Table 2). In Z-plasty cases, one hypertrophic scar was observed, and this was significantly resolved by two local triamcinolone injections. In V-Y advancement cases, the major complication was visible scaring, that is, as partial alopecia, caused by flap tension, and occurred at the apex of the advanced flap in seven cases. However, in almost cases, the visibility of scar due to alopecia became extinct by subsequent hair growth and the temporal hairline was lowered by inferior advancement of the triangular flap. Skin sloughing occurred in three cases and this was resolved by ointment dressing. However, healed area often tended to lose the hair, so that number of alopecia used to include cases of skin sloughing. There was one hypertrophic scar managed by triamcinolone injection, although simple suture scar existed in all cases. There were no other postoperative complications such as necrosis or wound infection.

Table 1. Postoperative complications

\begin{tabular}{lcc} 
Complication & $\begin{array}{c}\text { Z-plasty } \\
\text { (total } 9 \text { cases) }\end{array}$ & $\begin{array}{c}\text { V-Y advancement } \\
\text { (total 26 cases) }\end{array}$ \\
\hline Hypertrophic scar & $1(11.1)$ & $1(3.8)$ \\
Alopecia (Visible scar) & 0 & $7(26.9)$ \\
\hline Sloughing & 0 & $3(11.5)$ \\
Necrosis & 0 & 0 \\
\hline Infection & 0 & 0 \\
Total & $1(11.1)$ & $11(42.3)$ \\
\hline
\end{tabular}

Values are presented as number (\%).

Table 2. Management based on type and severity

\begin{tabular}{llccc}
\hline Type & Severity & V-Y advancement & Z-plasty & Number \\
\hline I & Mild & 9 & 9 & 18 \\
& Severe & 11 & 0 & 11 \\
$\|$ & Mild & 2 & 0 & 2 \\
& Severe & 4 & 0 & 4 \\
\hline \multirow{2}{*}{ Total cases } & & 26 & 9 & 35 \\
\hline
\end{tabular}

\section{DISCUSSION}

Many techniques have been introduced to correct cryptotia, since Kubo [8] first reported a surgical method. The main principles of surgical treatment are supplementation of skin deficiency and the correction of cartilage deformity. Constructing an auriculocephalic sulcus at the same time should be performed to obtain the required upper auricular area shape. A skin deficiency is often present in the upper auricular region, particularly of the posterior auricular surface. In addition, cartilage deformity related to abnormal muscle attachment is common and should be released for adequate correction [11].

The skin deficiency can be treated by releasing the upper pole and coverage using a transposition flap [12,13], preauricular flap [14], rotation flaps [2], Z-plasty [5-7], a subcutaneous pedicled flap [15], or a skin graft [16]. Of the various techniques available, the correction by V-Y advancement or Z-plasty was used.

In a previous report, we described cryptotia correction using V-Y advancement of a temporal triangular flap based on a modification of Kubo's method [8,9]. A large, long triangular flap prepared in the upper auricular region was advanced inferiorly to prepare the superior and posterior surfaces of the upper auricular half. The characteristics of V-Y advancement can be summarized as follows: 1) simple, easy design and a short operation time; 2) provision of enough skin to the upper and posterior portions of the auricle; 3) provision of sufficient depth of auriculocephalic sulcus; 4) correction of cartilage deformities with unrestricted access; 5) no need for additional skin grafting; 6) applicable for other ear deformities including constricted ear; 7) visible scarring at the donor site; and 8) lowered hairline created by advancement of the temporal triangular flap.

Flap adaptability with respect to the amount of skin deficiency is also an advantage of V-Y advancement, which means the technique can be applied to almost all cases by regulating the height of the triangular flap. In severe cases as deficiency amount, it is corrected by higher position of peak point of triangular flap, the height from superior helix may be 7 to $8 \mathrm{~cm}$. In mild cases, lower position can be permitted according to the amount of skin deficiency and the height may be 5 to $6 \mathrm{~cm}$. However, even when a small triangu- 
lar flap is used, the main disadvantage of V-Y advancement is a visible scar, which is inevitable, especially in men with short hair. As mentioned above, the disadvantages of $\mathrm{V}-\mathrm{Y}$ advancement are the development of a visible scar at the donor site (alopecia) and a lowered hairline created by advancement of the temporal triangular flap. The lowered hairline can be corrected using a depilation laser, but visible scarring cannot be corrected easily.

Thus, the correction of cryptotia using Z-plasty could be used, especially in mild cases. Z-plasty is designed on the periauricular area possibly extending to hair bearing scalp. The advantages of Z-plasty are similar to those of V-Y advancement, and are as follows: simple and easy technique, provision of enough skin to provide sufficient depth of auriculocephalic sulcus in cases of mild skin deficiency, correction of cartilage deformities with unrestricted access, and no need for additional skin grafting. However, the main advantage of Z-plasty is the low risk of visible scarring at the donor site, because this is located in the posterior and superior auricular area, and can be easily hidden and is less prominent. The scar line is almost invisible due to the corrected superior helix in the superior auricular area and the posterior scar line is at or slightly within the hairline. The flap area is adjusted according to needed amount of skin replacement, and that is posterior margin of the posterior based flap can be at hair line or about $1 \mathrm{~cm}$ inner area of hair line in proportion to the length of flap or existence of back cut line. In case of posterior margin inner area of hair line, of course, the hair line displacement can occurred. However, the displaced area is just small and can be easily corrected depilation laser on several times.

As mentioned above, the flap size was modified according to the amount of skin needed for replacement. For the Z-plasty technique, the width and length of the posterior flap can be adjusted based on considerations of extent of flap advancement, skin relaxivity and the severity of skin deficiency. The posterior based flap was usually modified on a case-by-case basis. When a large volume of skin flap is required, the lateral arm of the posterior based flap was incised from below the midlevel of the auricle to, but not exceeding, $1 \mathrm{~cm}$ within the hairline. In mild cases, the incision was placed at the hairline. If needed, a back-cut is performed on inferior edge of the lateral arm of posterior based flap. For V-Y ad- vancement, the height of the triangular flap was controlled. The usual position of the peak point of a triangular flap is 7 to $8 \mathrm{~cm}$ from the superior helix, but in mild cases, it is 5 to $6 \mathrm{~cm}$ from the superior helix, which reduces scarring. Thus, both Z-plasty and V-Y advancement offer flexibility with respect to the amount of skin deficiency and severity, which means flap adaptability.

The manipulation of cartilage and fibrous tissue is another important point that requires consideration. Cryptotia presents various chracteristics; deficiencies of soft tissue in the helix, deformity of auricular cartilage, contraction of the anterior and posterior lengths of cartilage, marked inward turning and adhesion of cartilage in the antihelix, and adhesion of helix cartilage to the scaphoid cavity. In order to achieve an adequate correction of cryptotia, it is necessary to dissect muscle at the superior and posterior surfaces of the antihelix cartilage and fibrous tissues when severe contracture of the antihelix is present $[2,14,17,18]$. Furthermore, individual cartilage deformities should be corrected on a case-bycase based on the severity of the deformity $[9,19]$. In our cases, the cartilage manipulation was also done by incision along the posterior aspect of the upper helix, flattening of antihelix using mattress sutures, scoring of the scapha, and by using Musgrave's technique. Either concha cartilage or Medpor sheet graft was used to prevent recurrence of cartilaginous deformities, as recommended by Onizuka et al. [6] and Kim et al. [20], respectively. It was applied to the posterior aspect of the crus of the antihelix to produce a splinting effect and maintaining effect [21]. We used a cartilage graft in 9 cases and Medpor sheet in 26 cases on the posterior aspect of the crus of the antihelix as a splint. It is believed that a Medpor sheet provides a firmer splinting effect than a cartilage graft, and thus, better prevents relapse. Some consider that Medpor sheet introduces risks of foreign body reaction, inflammation, and infection, but we did not experience any of these problems. Furthermore, its uses had several advantages, such as a short operative time, simplicity, and no donor site morbidity. Lee et al. [22] also recommended that Medpor sheet could be used for otoplasty because of its availability, shape maintaining properties, and lack of a need for a donor. Thus, Medpor sheet is considered to have many advantages as a graft material for the correction of cryptotia.

In summary, we used two methods, Z-plasty and V-Y advance- 
ment, to correct cryptotia, and decided which to use based on the severity of skin deficiency. The main advantage of Z-plasty is the low risk of visible scarring at the donor site. In mild cases, the correction of cryptotia using Z-plasty offers a good alternative solution, but in severe cases, V-Y advancement remains the preferred treatment option for more sufficient volume than Z-plasty.

The main advantage of Z-plasty is lower likelihood of visible scarring at the donor site. In mild cases, Z-plasty may be good alternative, but in severe cases, $\mathrm{V}-\mathrm{Y}$ advancement which supplies sufficient volume is probably the best option.

\section{REFERENCES}

1. Ohmori S, Matsumoto K. Treatment of cryptotia, using Teflon string. Plast Reconstr Surg 1972;49:33-7.

2. Hirose T, Tomono T, Matsuo K, Katohda S, Takahashi N, Iwasawa M, Satoh R. Cryptotia: our classification and treatment. Br J Plast Surg 1985;38:352-60.

3. Adams MT, Cushing S, Sie K. Cryptotia repair: a modern update to the trefoil flap. Arch Facial Plast Surg 2011;13:355-8.

4. Paredes AA Jr, Williams JK, Elsahy NI. Cryptotia: principles and management. Clin Plast Surg 2002;29:317-26.

5. Yotsuyanagi T, Yamashita K, Shinmyo Y, Yokoi K, Sawada Y. A new operative method of correcting cryptotia using large Z-plasty. Br J Plast Surg 2001;54:20-4.

6. Onizuka T, Tokunaga S, Yamada K. A method for repair of cryptotia. Plast Reconstr Surg 1978;62:734-8.

7. Sohn YH, Yang WS, Byun JS, Baek BS. Modified Onizuka’s method for correction of cryptotia. J Korean Soc Plast Reconstr Surg 1989;16:947-55.
8. Kubo I. Taschenohr und otoplastik. Oto-Rhino-Laryngology 1933;6:105-10.

9. Cho BC, Han KH. Surgical correction of cryptotia with V-Y advancement of a temporal triangular flap. Plast Reconstr Surg 2005;115:157081.

10. Musgrave RH. A variation on the correction of the congenital lop ear. Plast Reconstr Surg 1966;37:394-8.

11. Hodgson EL, McGregor AD. Correction of cryptotia using full-thickness skin grafts. Ann Plast Surg 2001;47:471-2.

12. Fukuda O. Otoplasty of cryptotia. Keisei Geka 1968;11:117-25.

13. Ono I, Gunji H, Suda K, Tateshita T, Kaneko F. A new operative method for treating severe cryptotia. Plast Reconstr Surg 1995;96:461-8.

14. Elsahy NI. An alternative technique for correction of cryptotia. Ann Plast Surg 1989;23:66-73.

15. Wesser DR. Repair of a cryptotic ear with a trefoil flap: case report. Plast Reconstr Surg 1972;50:192-3.

16. Nishimura Y. Correction of cryptotia (pocket ear). Jpn J Plast Reconstr Surg 1975;18:696-8.

17. Thorne CH, Wilkes G. Ear deformities, otoplasty, and ear reconstruction. Plast Reconstr Surg 2012;129:701e-16e.

18. Kajikawa A, Ueda K, Asai E, Ohkouchi H, Katsuragi Y. A new surgical correction of cryptotia: a new flap design and switched double banner flap. Plast Reconstr Surg 2009;123:897-901.

19. Cho BC, Lee JH, Choi KY, Yang JD, Chung HY. Fabrication of stable cartilage framework for microtia in incomplete synchondrosis. Arch Plast Surg 2012;39:162-5.

20. Kim DY, Cho KS, LeeSY, Cho BH. Surgical correction of cryptotia using Medpor. Ann Plast Surg 1999;42:693-9.

21. Kim SK, Yoon CM, Kim MH, Kim MS, Lee KC. Considerations for the management of cryptotia based on the experience of 34 patients. Arch Plast Surg 2012;39:601-5.

22. Lee KC, Kwon YS, Heo J, Moon JB, Kim SK. Otoplasty with high density polyethylene implant (MEDPOR(R)). J Korean Soc Plast Reconstr Surg 2009;36:167-73. 